\title{
COMPUTED TOMOGRAPHIC FINDINGS IN 35 DOGS WITH NASAL ASPERGILLOSIS
}

\author{
Jimmy H. Saunders, DVM, Jean-Luc Zonderland, DVM, Cécile Clercx, DVM, PhD, \\ Ingrid Gielen, DVM, MS, Frédéric R. SnaPs, DVM, PhD, Martin Sullivan, DVM, PhD, \\ Henri vanBree, DVM, PhD, Robert F. Dondelinger, DVM, PhD
}

\begin{abstract}
The purpose of this study was to describe the computed tomographic (CT) features of nasal aspergillosis in dogs. Initial $(n=35)$ and follow-up $(n=12)$ CT images were available from 35 dogs. The most commonly encountered $\mathrm{CT}$ findings were (1) moderate to severe cavitary destruction of the turbinates with presence of a variable amount of abnormal soft tissue in the nasal passages, (2) non-specific thickening of the mucosa adjacent to the inner surface of bones of the frontal sinus, maxillary recess and nasal cavity and, (3) thickened reactive bone. The findings were consistent with a disease initially affecting one nasal cavity then progressing into the ipsilateral frontal sinus, the contralateral nasal cavity and the contralateral frontal sinus. Two dogs with associated nasal foreign body had a more localized invasion of the nasal cavity. Attenuation values and contrast enhancement were not specific. With follow-up examinations, a reduction in the amount of abnormal soft tissue was observed in all dogs except one, but this reduction could not be quantified. Veterinary Radiology \& Ultrasound, Vol. 43, No. l. 2002 pp 5-9.
\end{abstract}

Key words: aspergillosis, canine, computed tomography, nasal cavity.

\section{Introduction}

$\mathrm{F}$ UNGAL RHINITIS is a common disease in dogs and accounts for 7 to $34 \%$ of dogs with chronic nasal disease. ${ }^{1-3}$ Aspergillus fumigatus is the most frequently isolated organism in canine nasal and paranasal sinus infections. ${ }^{4}$ In most dogs, the disease tends to be invasive and slowly destroys the turbinates. ${ }^{5}$

Diagnosis is established with help of clinical history, physical examination, radiography, computed tomography (CT), rhinoscopy, serology, cytology, histology, and mycologic culture. ${ }^{5,6}$ Conventional radiography permits differentiation between nasal neoplasia and nasal aspergillosis in the majority of dogs. ${ }^{7}$ The main radiologic features of nasal aspergillosis are turbinate loss with punctate lucencies of the supporting bones, increased radiolucency rostrally and increased opacity caudally in the nasal cavity, and increased

From the Department of Medical Imaging, Faculty of Veterinary Medicine. Ghent University, Salisburylaan 133, 9820 Merelbeke, Belgium (Saunders, Gielen, vanBree); Departments of Small Animals (Zonderland, Clercx) and Medical Imaging (Snaps), Faculty of Veterinary Medicine, University of Liège, Belgium; Department of Veterinary Clinical Studies, University of Glasgow, Scotland (Sullivan); Department of Medical Imaging, University Hospital Center, University of Liège, Belgium (Dondelinger).

Address correspondence and reprint requests to Jimmy H. Saunders, Department of Medical Imaging, Faculty of Veterinary Medicine, Ghent University, Salisburylaan 133, 9820 Merelbeke, Belgium. Email address: Jimmy.Saunders@rug.ac.be

Received September 20, 2000; accepted for publication July 28, 2001. opacity in the frontal sinus with thickened and mottled frontal bone. ${ }^{8}$

CT is a reliable, non-invasive technique for the evaluation of chronic nasal disease in dogs and is also useful to define the extension of lesions for radiation therapy planning. ${ }^{9-11} \mathrm{CT}$ is superior to radiography for identification of unilateral vs. bilateral nasal cavity disease and to define the extension of lesions into adjacent structures. ${ }^{11,12}$ However, to date there is no detailed study about the specific CT features of canine nasal aspergillosis. The aim of our study is to describe the pre- and post-treatment CT features of nasal aspergillosis in dogs and to relate these to the spread of the disease.

\section{Materials and Methods}

The CT images of 35 dogs with nasal aspergillosis seen at the Department of Medical Imaging, Ghent University $(\mathrm{n}=11)$ and the Small Animals Clinic, University of Liège ( $\mathrm{n}=24$ including all follow-up scans) between August 1997 and January 2000 were used for this study. Age ranged from 9 months to 10 years (mean age $=4.3$ years). Fifteen different breeds were represented (8 Rottweiler, 5 Labrador Retriever, 5 Golden Retriever, 3 Belgian Shepherd dogs, 2 Doberman, 2 Afghan Wolfhound, 2 mixed breed dogs, 1 Newfoundland, 1 Alaskan Malamute, 1 Basset Hound, 1 Dachshund, 1 Bull Terrier, 1 German Shepherd dog, 1 Pyrenean Shepherd, 1 Bobtail). There were 20 males and 15 females. 
A physical examination was performed on each dog. Then, anesthesia was induced with droperidol + fentanyl* at a dose of $0.08 \mathrm{mg} / \mathrm{kg}$ body weight IV, penthotal $†$ at a dose of $5-15 \mathrm{mg} / \mathrm{kg}$ body weight IV and maintained with halothane $\neq$ (1.5-2\%). Radiographic, CT and rhinoscopic examinations were performed during this anesthetic procedure. Swabs, cytobrush, and biopsies were taken during rhinoscopy for mycologic culture, cytology, and histology. Blood samples were also taken for serology. Previously established guidelines were used to consider a definite diagnosis. $^{13}$

CT examinations were performed with either a $3^{\text {rd }}$ generation conventional CT scanner $\$$ at the Ghent University $(120 \mathrm{kV}, 100 \mathrm{~mA}$, slice thickness $2 \mathrm{~mm})$ or a $4^{\text {th }}$ generation helical CT scanner" at the University of Liège $(110 \mathrm{kV}, 125$ $\mathrm{mA}$, pitch 1.5 , index 3 , slice thickness $3 \mathrm{~mm}$ ) with the dogs in ventral recumbency. Transverse slices were acquired from the caudal level of the frontal sinus to the nostrils. Preand post-contrast examinations using a bolus IV injection of $700 \mathrm{mg} / \mathrm{kg}$ of body weight of non-ionic iodine contrast medium\# were performed. Reformatted dorsal plane images were also obtained. Regions of interest (ROI) were drawn on mucosal thickening and on abnormal soft-tissues in the nasal cavity and/or frontal sinus and, time-density attenuation data were measured before and after contrast medium administration.

The CT criteria that were evaluated were based on a previous study, ${ }^{14}$ complemented with our experience. Major CT criteria were: extension of the lesions (rostral, middle, caudal nasal cavity, frontal sinus, uni- or bilateral); presence or absence of abnormal soft-tissues and/or mucosal thickening (nasal passages classified as absent $=$ no abnormal soft tissue-mild $=$ abnormal soft tissue in $1 / 3$ of the nasal cavity-moderate $=2 / 3$ or severe $=$ all, maxillary recesses, caudal recesses, retrobulbar space, pharynx, frontal sinuses, presence of a focal rounded soft tissue accumulation); presence of lysis (cribriform plate, turbinates classified as absent $=$ no turbinate lysis - $\mathrm{mild}=$ turbinate lysis in $1 / 3$ of the nasal cavity-moderate $=2 / 3$ or severe $=$ all, maxillary recesses, bones associated with the caudal recesses, nasal, frontal, palatine, ethmoid, maxillary and vomer bones) and presence of hyperostosis or reactive new bone. A pattern was defined as generalized when the entire nasal cavity and frontal sinus were affected on at least one side and as localized when restricted to the nasal passage.

Hard copies were printed with a bone window (window width (WW) 3500-window level (WL) 500) and a softtissue window (WW 340-WL 25) for each dog. These

\footnotetext{
*Thalamonal ${ }^{\circledR}$, Janssen-Cilag, Beerse, Belgium.

†Phenobarbital ${ }^{\oplus}$, Abbott, Illinois.

$\$$ Fluothane ${ }^{\text {iit }}$, Zeneca, Delaware.

\$Pace CT, GE Medical Systems, Milwaukee.

"Picker 6000, Picker International Inc., Ohio.

\#Omnipaque 300, Nycomed, Brussels, Belgium.
}

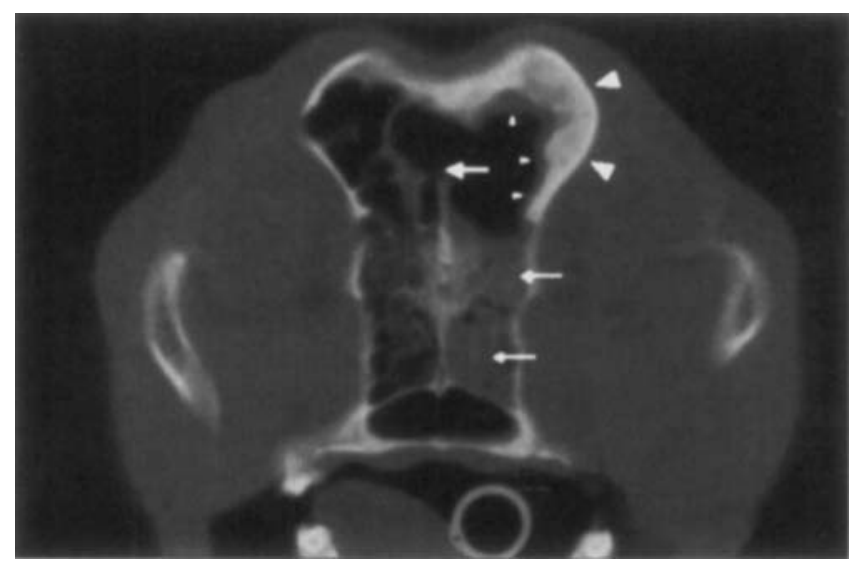

FIG. 1. Transverse image ( window width $=3500$, window level $=500$ ) of the frontal sinus and nasal cavity at the level of the second maxillary premolar of a 12-year-old rottweiler. There is hyperostosis of the lateral wall of the left frontal sinus (large arrowheads) with mucosal thickening along this inner surface of the bone (small arrowheads). Lysis of the frontal crest (large arrow) is also present. Presence of abnormal soft-tissue is noted in the left the nasal cavity (small arrows).

copies were reviewed jointly by two of the authors (JHS, FRS). When necessary, the WW and WL were adjusted on the computer monitor for visualization of other structures.

All dogs were treated with a noninvasive intranasal infusion of enilconazole $1 \%$.** The treatment was repeated each 6 weeks until there was no clinical or rhinoscopic evidence of infection. The follow-up images were acquired one month after clinical resolution with the same scanning protocol and were reviewed using the same methodology as used previously. Special attention was given to potential regrowth of the conchae, bone healing and effect of sampling on turbinate appearance.

\section{Results}

The lesions were generalized in 26 dogs $(26 / 35=74 \%)$ with the contralateral side affected in 19 dogs $(19 / 26=$ $73 \%)$. The lesions were localized in 9 dogs $(9 / 35=25 \%)$ with the contralateral side affected in 4 dogs $(4 / 9=44 \%)$. Lesions were bilateral in 23 of the 35 dogs $(23 / 35=65 \%)$.

Frontal sinus abnormalities were seen in $26 \mathrm{dogs}(26 / 35$ $=74 \%$ ) and included mucosal thickening (Fig. 1) in 19 dogs $(19 / 26=73 \%)$, complete obliteration of the sinus by soft tissue in 4 dogs $(4 / 26=15 \%)$ and presence of abnormal soft tissue without mucosal thickening in 3 dogs $(3 / 26$ $=11 \%$ ). In addition, the presence of a large amount of abnormal soft tissue associated with the sino-nasal ostium was noted in $24 \operatorname{dogs}(24 / 26=92 \%)$. The frontal bone was affected in 24 dogs $(24 / 26=92 \%)$, unilaterally in 13 dogs $(13 / 24=54 \%)$ and bilaterally in $11 \mathrm{dogs}(11 / 24=46 \%)$. In 10 of these 11 dogs, there was frontal crest lysis and extension to the contralateral side (Fig. 1). The frontal bone

**Imavérol ${ }^{\circledR}$, Janssen-Cilag, Beerse, Belgium. 
pattern was predominantly hyperostotic in 10 dogs $(10 / 24$ $=42 \%)$, predominantly lytic in $2 \operatorname{dogs}(2 / 24=8 \%)$ and mixed in $12 \operatorname{dogs}(12 / 24=50 \%)$.

Nasal passage abnormalities (Fig. 2) were present in all 35 dogs $(35 / 35=100 \%)$. On the most affected side, the turbinate destruction was severe in $26 \operatorname{dogs}(26 / 35=74 \%)$, moderate in 8 dogs $(8 / 35=23 \%)$ and mild in $1 \mathrm{dog}(1 / 35$ $=3 \%$ ). On the less affected side, the turbinate destruction was severe in 2 dogs $(2 / 35=5 \%)$, moderate in 7 dogs $(7 / 35$ $=20 \%)$, mild in 10 dogs $(10 / 35=29 \%)$ and absent in 16 dogs $(16 / 35=46 \%)$. On the most affected side, the amount of abnormal soft tissue was severe in $15 \mathrm{dogs}(15 / 35=$ $43 \%)$, moderate in 9 dogs $(9 / 35=26 \%)$ and mild in 11 dogs $(11 / 35=31 \%)$. On the less affected side, it was severe in 2 dogs $(2 / 35=5 \%)$, moderate in 5 dogs $(5 / 35=$ $15 \%)$, mild in 10 dogs $(10 / 35=29 \%)$, and absent in 18 $\operatorname{dogs}(18 / 35=51 \%)$. Cribriform plate destruction and replacement of fatty retrobulbar tissue with soft tissue were present in 2 dogs $(2 / 35=5 \%)$. Abnormal soft tissue was present in the caudal recesses in $15 \operatorname{dogs}(15 / 35=42 \%)$ and bony lesions in 5 dogs $(5 / 35=14 \%)$. For the maxillary recesses, mucosal thickening and abnormal soft tissue were observed in 14 dogs $(14 / 35=40 \%)$ and a mucosal thickening alone in $10 \operatorname{dogs}(10 / 35=28 \%)$. Bone lysis of the ethmoid bone (orbital lamina) was visible in $17 \mathrm{dogs}(17 / 35$ $=48 \%)$. The maxillary bone was affected in 16 dogs $(16 / 35$ $=45 \%)$. The nasal septum was deviated in 7 dogs $(7 / 35=$ $20 \%)$, destroyed in 8 dogs $(8 / 35=23 \%)$ or both in 11 dogs $(11 / 35=31 \%)$. Lysis of the vomer bone was present in 11 dogs $(11 / 35=31 \%)$ and of the nasal bone in 2 dogs $(2 / 35$ $=5 \%$ ). On dorsal and/or sagittal reconstructions a cavitating process was present in all dogs, even apparent in dogs with a severe accumulation of soft tissue $(35 / 35=100 \%)$ (Fig. 3). The term cavitating process describes an aggressive lytic pattern where the affected turbinates are completely destroyed leaving a homogeneously black empty air filled space. An extramucosal mass-like soft tissue accumulation could be distinguished in 2 dogs $(2 / 35=5 \%)$.

Follow-up examinations in dogs having clinical resolution were characterized by a decrease in soft-tissue in 11 of the 12 follow-up examinations $(11 / 12=91 \%)$ including a reduction of the mucosal thickening in the nasal cavity in 6 dogs $(6 / 12=50 \%)$. Bony changes, conchae regrowth or changes related to sampling were not observed.
A

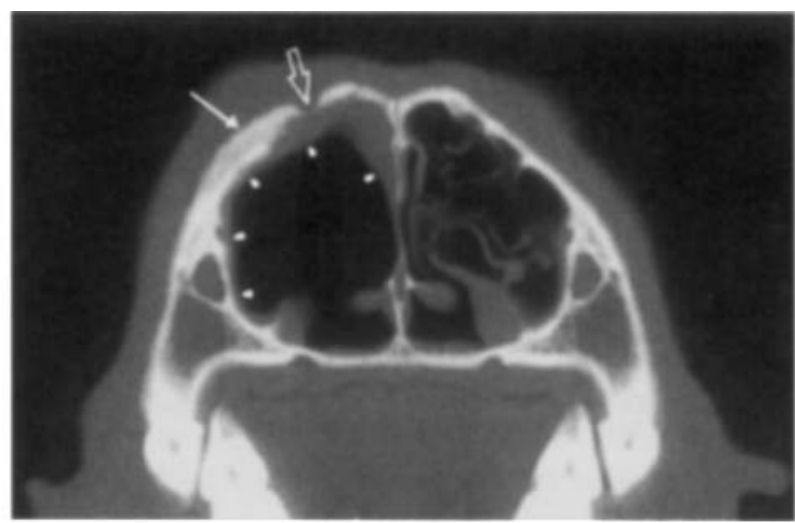

C

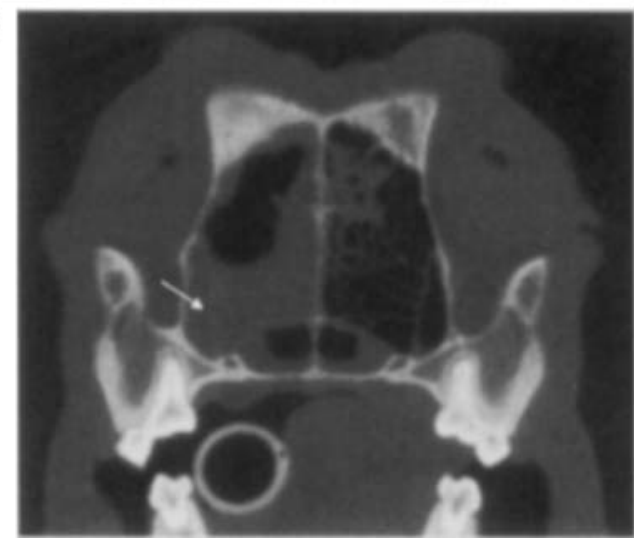

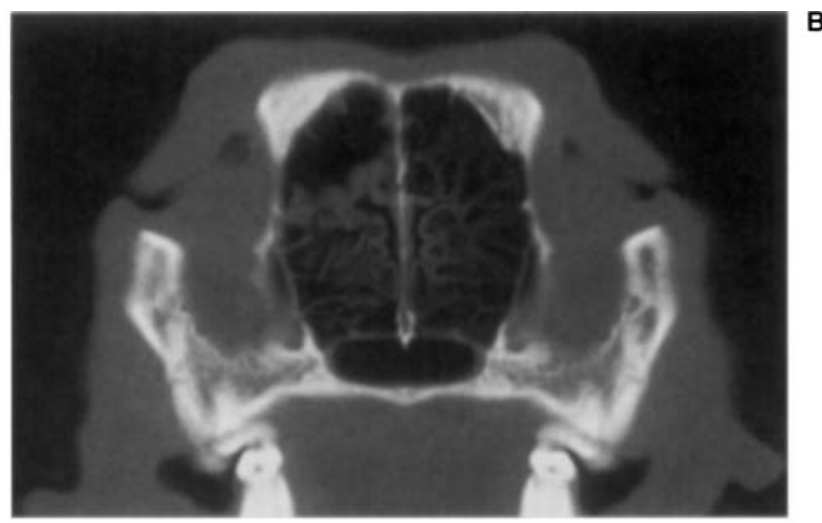

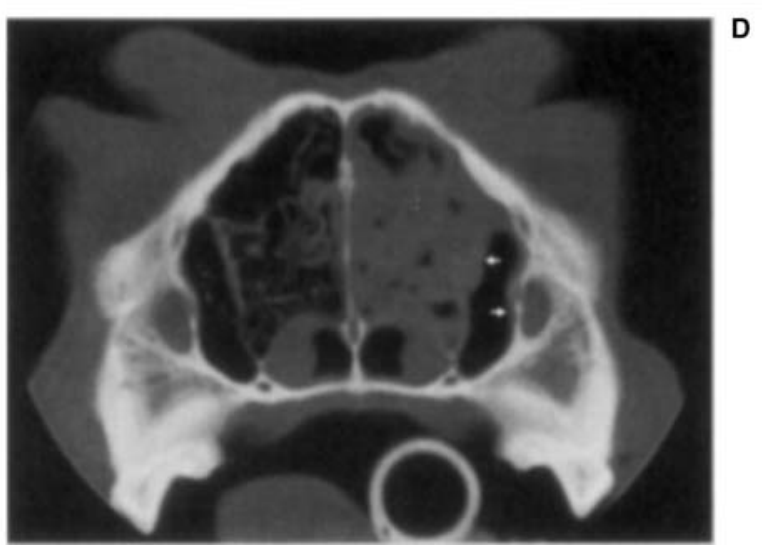

FIG. 2. Transverse images (window width $=3500$, window level $=500$ ) of the nasal cavity illustrating the different patterns that may be encountered in dogs with nasal aspergillosis. (A) Complete turbinate lysis, mucosal thickening (arrowheads) and, maxillar bone hyperostosis (arrow) and lysis (open arrow) in the right nasal cavity. (B) Localized turbinate lysis, thickening and distortion of the turbinates in the right nasal cavity. (C) Turbinate lysis dorsally, mucosal thickening, abnormal soft tissue and complete filling of the maxillary recess (arrow) in the right nasal cavity. Abnormal accumulation of soft tissue densities are also visible in the left nasal cavity. (D) Localized turbinate lysis (bilaterally), severe amount of abnormal soft-tissue and mucosal thickening in the left maxillary recess (arrows) in the left nasal cavity. 


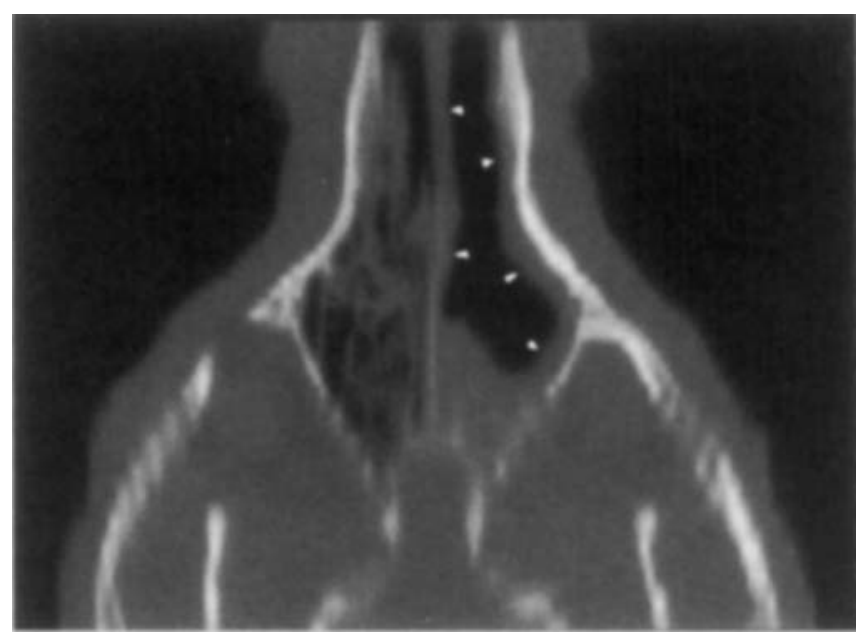

FIG. 3. Dorsal image, reformatted, of a 5-year-old rottweiler. There is destruction of the turbinates and mucosal thickening (arrowheads) in the left nasal cavity.

Reliable attenuation values could not be measured in the nasal cavity, except when the ventral nasal conchae were present $(n=12)$. These conchae had pre- (mean 74.1 HU $\pm 21.5 \mathrm{HU}$ ) and post contrast (mean $230.4 \mathrm{HU} \pm 87.5 \mathrm{HU}$ ) values comparable to normal and other pathologic nasal tissue.

The abnormal mucosal thickening in the frontal sinus ( $\mathrm{n}$ $=19)$ had pre-contrast attenuation values between 10 and $70 \mathrm{HU}$ (mean $37.1 \mathrm{HU} \pm 20.9 \mathrm{HU}$ ) and was not enhanced.

\section{Discussion}

There is no specific study of the CT features of canine nasal aspergillosis. However, a few studies about chronic canine nasal disease included dogs with aspergillosis. ${ }^{6,14,21}$ Unilateral loss of turbinates, local nasal mucus accumulation and regional mucosal swelling were described. ${ }^{6,14,21}$ In another study on 12 dogs with nasal aspergillosis, turbinate destruction, and paranasal fluid accumulations were evident. ${ }^{22}$ A cavitating lesion was again a significant feature. ${ }^{22}$ In our study, the most common CT findings were: (1) moderate to severe destruction of the turbinates with a variable amount of abnormal soft tissue in the nasal passages; (2) non-specific thickening of the mucosa along the bones of the frontal sinus, maxillary recess and nasal cavity; and (3) thickened reactive bone (reactive bone dorsolaterally and localized frontal crest lysis). It should be noted that the cavitated appearance, due to turbinate lysis, was still detectable in dogs with a large amount of abnormal soft tissue in the nasal passages. When the frontal sinus was involved, there was mostly a severe amount of abnormal soft tissue around the sino-nasal ostium.

No dog had a frontal sinus infection without a concurrent abnormality in the nasal cavity. This finding suggests that aspergillosis in these dogs presented as an aggressive (de- structive), slowly progressive process, affecting the nasal turbinates of one nasal cavity, then extending into the contralateral nasal cavity and/or ipsilateral frontal sinus and finally via frontal crest lysis into the contralateral frontal sinus.

Because of its cross-sectional images, CT has been proven to be superior to conventional radiography in demonstrating the extent of lesions and in differentiating infectious rhinitis and nasal neoplasia in both dogs, cats and humans. ${ }^{11,15,16}$ Dorsal reconstructions permit accurate assessment of the integrity of the cribriform plate. ${ }^{17}$ Dorsal reconstructions were also the most appropriate to assess the full extension of the cavitating process in this study.

Eleven examinations were obtained with a conventional CT and 24 with a helical CT. The advantages of helical vs conventional CT are a significantly reduced acquisition time with consequent optimization of dynamic contrast studies, improved detection of solitary lesions and the quality of post-processing 2 - and 3-D reconstructions. ${ }^{18-20}$ In this study, the authors found no appreciable difference in image quality or reconstructions.

In man, CT permits differentiation between the aerogenic and dental forms of sinonasal aspergillosis. ${ }^{15}$ Imaging characteristics suggestive of fungal sinusitis are non-specific soft tissue changes (rim of soft-tissue attenuation of varying thickness along the bone) in the sinus with reactive bone and localized areas of osteomyelitis, and the association of inflammatory sinus disease with involvement of the adjacent nasal cavity. ${ }^{23,24}$ In CT densitometric examinations, organic masses containing calcium phosphate or carbonate can be seen. These represent endogenous fungal products, which indicate an aerogenic pathogenesis of sinusal aspergillosis. ${ }^{15}$ Such calcified masses were not observed in our dogs.

Attenuation measurements are susceptible to a variety of errors, particularly in a pathologic nasal cavity that comprises many complex structures of different physical densities (bone, cartilage, mucosa, air). ${ }^{25}$ In this study, measurements of attenuation values in the nasal cavity were not reliable, with the exception of the ventral nasal conchae where pre- and post-contrast attenuation values comparable to that measured in normal nasal cavity or in nasal tumors were found. ${ }^{11}$ This is in agreement with previous findings, and may be explained by the high vascularity even in normal nasal tissue. ${ }^{11}$

Attenuation values in the frontal sinuses were between 10 and $70 \mathrm{HU}$, corresponding to the measurements in humans with sinusal aspergillosis. ${ }^{26,27}$ A differentiation between mucoid secretions and mycetoma could not be made on the basis of the attenuation values. In man, the attenuation values of mucoid secretions vary with time from 10-25 HU for acute secretions to 30-60 HU for chronic secretions and the $\mathrm{HU}$ of a mycetoma falls within the same range making definite distinction impossible. ${ }^{27}$ Two extramucosal 
masslike soft tissue accumulations observed on rhinoscopy could not be found on subsequent CT examination. Contrary to observations made in the nasal cavity, no degree of enhancement of the mucosal thickening was observed in the frontal sinus. The authors suggest that the absence of enhancement may be caused by hypertrophic and/or hyperplastic, and fibrotic changes of the sinusal mucosa.

A possible etiopathogenesis of aspergillosis was not established but it was associated with foreign bodies in two dogs. An impacted canine tooth in a 10-month-old Belgian Shepherd dog and a bullet in a 9-month-old Labrador Retriever. Foreign bodies have been described as potential predisposing factors to nasal aspergillosis, but without supportive evidences. ${ }^{28}$

The characteristic CT features of canine nasal aspergillosis noted in this study were not typical of neoplasia. The CT findings with nasal neoplasia are patchy areas of increased soft tissue opacity, destruction of part of all of the ethmoid bone, abnormal soft tissue in the retrobulbar spaces, destruction of one or both of the lateral maxillae, destruction of the nasal bone or the rostral dorsal maxillae, or hyperostosis of the lateral maxilla. ${ }^{11,14}$

With follow-up examinations, a reduction in the amount of abnormal soft tissue was observed in all dogs except one, but this reduction could not be quantified. Reduction of mucosal thickening was present in half of the dogs in the nasal cavity but not in the frontal sinus. Regrowth of the conchae, bone healing or resolution of bone lysis were not observed. However, these changes require a long time to occur while our follow-up examinations were performed one month after clinical resolution.

To conclude, the CT findings most commonly encountered in nasal aspergillosis in dogs include cavitated-like turbinate lysis, a rim of soft tissue of variable thickness along bones in the frontal sinus, maxillary recess and nasal passages, the abnormal presence of soft tissue accumulation and thickened, reactive bony changes. Nasal aspergillosis should be considered a primary differential diagnosis when cavitated-like lysis and diffuse thickening of the nasal mucosa along the bones of the nasal cavity and frontal sinus are present.

\section{REFERENCES}

1. Lane JG, Clayton-Jones DG, Thoday KL, Thomsett LR. The diagnosis and successful treatment of Aspergillus Fumigatus infection of the frontal sinuses and nasal chambers of the dog. J Small Anim Pract 1974; 15:79-83.

2. Harvey CE, O’Brien JA. Nasal aspergillosis-penicilliosis. In Kirk RW (ed): Current Veterinary Therapy. WIII. Philadelphia, WB Saunders, 1983, pp 236-240.

3. Tasker S, Knottenbelt CM, Munro EAC, Stonehewer J, Simpson JW, Mackin AJ. Aetiology and diagnosis of persistent nasal disease in the dog: a retrospective study of 42 cases. J Small Anim Pract 1999;40:473-478.

4. Sharp NJH, Harvey CE, Sullivan M. Canine nasal aspergillosis and penicilliosis. Comp Cont Educ 1991;13:41-48.

5. Sharp NJH. Aspergillosis and Penicilliosis. In Greene GE (ed): Infectious diseases of the dog and cat. $2^{\text {nd }}$ ed. Philadelphia, WB Saunders, 1998, pp 404-409.

6. Colas G. Pathologie des cavités nasales et sinusales chez le chien et le chat. Prat Med Chir Anim Comp 1996;31:479-499.

7. Sullivan M, Lee R, Skae CA. The radiological features of sixty cases of intra-nasal neoplasia in the dog. J Small Anim Pract 1987;28:575-586.

8. Sullivan $M$, Lee R, Jakovljevic $S$, Sharp NJH. The radiological features of aspergillosis of the nasal cavity and frontal sinuses in the dog. J Small Anim Pract 1986;27:167-180.

9. Thrall DE, McLeod DA, Bentel GC, Dewhirst MK. A review of treatment planning and dose calculation in veterinary radiation oncology. Vet Radiology 1989;30:194-221.

10. McEntee MC, Page RL, Heidner GL, Cline JM, Thrall DE. A retrospective study of 27 dogs with intranasal neoplasms treated with cobalt radjation. Vet Radiology 1991;32:135-139.

11. Codner EC, Lurus AG, Miller JB, Gavin PR, Gallina A, Barbee DD. Comparison of computed tomography with radiography as a noninvasive diagnostic technique for chronic nasal disease in dogs. J Am Vet Med Assoc 1993;202:1106-1110.

12. Thrall DE, Robertson ID, McLeod D. Heidner GL, Hoopes PJ, Page LR. A comparison of radiographic and computed tomographic findings in 31 dogs with malignant nasal cavity tumors. Vet Radiology 1989;30:59-66.

13. Sharp N, Sullivan M, Harvey CE. Treatment of canine nasal aspergillosis. In Practice 1992;14:27-31.

14. Burk RL. Computed tomographic imaging of nasal disease in 100 dogs. Vet Radiology \& Ultrasound 1992;33:177-180.

15. Krenmair $\mathrm{G}$, Lenglinger $F$. Maxillary sinus aspergillosis: Diagnosis and differentiation of the pathogenesis based on computed tomography densitometry of sinus concretions. Journal of Oral Maxillo-Facial Surgery 1995;53:657-663.

16. Forrest LJ. The head: excluding the brain and orbit. Clin Techn Small Anim Pract 1999;14:170-176.

17. Koblik PD, Berry CR. Dorsal plane computed tomographic imaging of the ethmoid region to evaluate chronic nasal disease in the dog. Vet Radiology 1990;31:92-97.

18. Bonaldi VM, Garcia P, Coche EE, Sarazin L, Bret PM. Spiral computed tomography: a new CT imaging modality. La Presse Médicale 1996; 25:1109-1114.

19. Ney DR, Fishman EK, Kawashima A, Robertson DD, Scott WW. Comparison of helical and serial CT with regard to three-dimensional imaging of musculoskeletal anatomy. Radiology 1992;185:865-869.

20. Remy-Jardin M, Rémy J, Giraud F, Marquette $\mathrm{CH}$. Pulmonary nodules: detection with thick-section spiral CT versus conventional. Radiology 1993;187:513-520.

21. Schwarz T. Comparison of sensitivity and specificity of conventional X-ray and computed tomography (CT) in nasal tumors and mycoses in dogs. Vet Radiology 1995;36:428.

22. Mathews KG, Koblik PD, Richardson EF, Davidson AP, Pappagianis D. Computed tomographic assessment of noninvasive intranasal infusions in dogs with fungal rhinitis. Vet Surg 1996;25:309-319.

23. Centeno RS, Bentson JR, Mancuso AA. CT. scanning in rhinocerebral mucormycosis and aspergillosis. Radiology 1981;140:383-389.

24. Zinreich SJ, Kennedy DW, Malat J, Curtin HD, Epstein JI, Huff LC, Kumar AJ, Johns ME, Rosenbaum AE. Fungal sinusitis: diagnosis with CT and MR imaging. Radiology 1988;169:439-444.

25. Williams G, Bydder GM, Kreel L. The validity and use of computed tomography attenuation values. Britisch Med Bull 1980;36:279-287.

26. Fellows DW, Zinreich SJ. The paranasal sinuses and nasal cavity. In: Lee SH, Rao KCVG, Zimmerman RA ed. Cranial MRI and CT. $4^{\text {th }}$ ed. McGraw-Hill, New York. 1999; pp. 831-836.

27. Som PM, Curtin HD. Chronic inflammatory sinonasal diseases including fungal infections: the role of imaging. Radiol Clinics North America 1983;31:33-44.

28. Suter PF. Diseases of the nasal cavity, larynx and trachea. In: Suter PF ed. Thoracic radiography. A text atlas of thoracic diseases of the dog and cat. Wettswil, Switzerland. 1985; pp 206-223. 